\title{
AN OPEN MAPPING APPROACH TO HURWITZ'S THEOREM
}

\author{
BY \\ G. T. WHYBURN
}

1. Introduction. It is a consequence of known results $\left({ }^{1}\right)$ that any compact light open mapping $f$ of one two-dimensional manifold $A$ into another one $B$ has a definite finite degree $k(f)$, that is, there is an integer $k(f)$ such that for each $y \in f(A), f^{-1}(y)$ contains exactly $k(f)$ points provided the points of $f^{-1}(y)$ are counted with multiplicity in an obvious manner. Further, considered as a function on the subset $O$ of compact light strongly open mappings in the mapping space $B^{A}, k(f)$ can be proven lower semi-continuous for very much broader classes of spaces $A$ and $B$. Upper semi-continuity of $k(f)$ presents considerably more difficulty and requires restrictions on $A$ and $B$ as indicated. However, results will be established below from which it follows that $k(f)$ is indeed continuous on the set $O$ of compact light strongly open mappings of $A$ into $B$ where strongly open means that the image of every open set in $A$ is open in $B$ and not merely open in $f(A)$ (or, equivalently, $f$ is an open mapping and $f(A)$ is an open set in $B$ ).

As a consequence of our main theorem, we obtain a conclusion concerning the equality of the number of " $y$-places" in a given region of two "sufficiently close" light open mappings which is closely related to the classical theorems of Rouché and Hurwitz on the zeros of analytic functions and indeed which includes the Hurwitz theorem in its full strength as a special case. Thus, not only do we have a purely topological approach to this basic result of analysis, but one which leans entirely on the properties and techniques of open mappings and does not even involve any homotopy results or methods. As would be expected, the Hurwitz theorem can also be obtained by means of homotopy results and by using the concept of degree of a mapping defined in terms of homology or cohomology group mappings. The major difficulty in this latter is encountered in showing that, in the cases under consideration, the two types of degree are numerically the same. This and other related questions will be considered in a later paper.

Usually our spaces $A$ and $B$ are assumed to be locally connected generalized continua, that is, separable, metric, locally compact, connected, and locally connected. Any different conditions on the spaces will be clearly

Received by the editors September 30, 1950.

(1) For this and other results and definitions used concerning open mappings the reader is referred to the author's paper, Open mappings on locally compact spaces, Memoirs of the American Mathematical Society, no. 1, 1950 and his book, Analytic topology, Amer. Math. Soc. Colloquium Publications, vol. 28. See also, Stoilow, Leçons sur les principes topologiques de la theorie des fonctions analytiques, Gauthier-Villars, 1938. 
indicated in the statements of the results. A region $R$ in a space $A$ is a connected open subset of $A$ and the boundary $\bar{U}-U$ of any open set $U$ will be denoted by $\operatorname{Fr}(U)$. For any set $X$ and $r>0, V_{r}(X)$ denotes the set of all points $x$ satisfying $\rho(x, X)<r$. All mappings are assumed continuous and single-valued. A mapping $f: A \rightarrow B$ is compact provided that for any compact set $K \subset B, f^{-1}(K)$ is compact and is light provided that $f^{-1}(y)$ is 0 -dimensional for each $y \in B$.

2. TheOREM. Let $A$ and $B$ be separable metric spaces, let $f$ be any mapping of $A$ into $B$ and let $R$ be any set in $B$. For any nonempty conditionally compact component $Q$ of $f^{-1}(R), f: Q \rightarrow R$ is a compact mapping.

Proof. Denote $f \mid Q$ by $g$ and let $K$ be any compact set in $R$. Then since $f^{-1}(K)$ is closed and $\bar{Q}$ is compact, $\bar{Q} \cdot f^{-1}(K)$ is compact. But $\bar{Q} \cdot f^{-1}(K)=Q$ $\cdot f^{-1}(K)=g^{-1}(K)$ since $(\bar{Q}-Q) \cdot f^{-1}(K) \subset(\bar{Q}-Q) \cdot f^{-1}(R)=0$.

Corollary. If $A$ and $B$ are locally connected generalized continua, $f$ is strongly open, $R$ is a region in $B$, and $Q$ is a conditionally compact component of $f^{-1}(R)$, then $f(Q)=R$ and $f \mid Q$ is compact.

For since $Q$ is open in $A, f(Q)$ is both open and closed in $R$ and thus is equal to $R$.

3. Theorem. Given a mapping $f: A \rightarrow B$, where $A$ and $B$ are locally connected generalized continua, any continuum (compact!) $H \subset B$ and any conditionally compact open set $U \subset A$ such that $U \cdot f^{-1}(H) \neq 0, \operatorname{Fr}(U) \cdot f^{-1}(H)=0$. Then there exists a $d(H)>0$ such that if $g: A \rightarrow B$ is strongly open and satisfies $\rho(f, g)<d(H)$, then $g(U) \supset H$ and $U \cdot g^{-1}(H)$ is compact.

Proof. Let $e=\rho[H, f(C)] / 2$ where $C=\operatorname{Fr}(U)$, let $R$ be the component of $V_{e}(H)$ containing $H$, and let $d(H)=\rho[H, B-R]$. Then if $g$ satisfies our conditions, we have $g\left[f^{-1}(H)\right] \subset R$ and $g(C) \subset B-R$ (since $d(H) \leqq e$ ). Thus if $Q$ is any component of $g^{-1}(R)$ intersecting $U$, we have $Q \subset U$ since $C \cdot g^{-1}(R)=0$. Accordingly $Q$ is conditionally compact and $g(Q)=R \supset H$; and $U \cdot g^{-1}(H)$ is compact since $C \cdot g^{-1}(H)=0$.

CoRollary 1. Any such mapping $g$ is a compact mapping on the set $U \cdot g^{-1}(R)$.

Corollary 2. If $f$ is a compact mapping on $A, d(H)>0$ may be chosen so that if $\rho(f, g)<d(H), g$ is a compact mapping on $g^{-1}(H)$.

For $U$ may be chosen so as to contain $f^{-1}(H)$, since this set is compact; and $d(H)$ may also be taken less than $\rho[H, f(A-U)]$, since $f(A-U)$ is a closed set not intersecting $H$. This gives $g^{-1}(H) \subset U$ so that $g^{-1}(H)$ is compact. 
COROLlaRY 3. If $U$ contains at least $k$ components of $f^{-1}(H)$, there exists a $d(H)>0$ such that for $\rho(f, g)<d(H), U$ contains at least $k$ distinct components of $g^{-1}(H)$.

For we may choose disjoint open sets $U_{1}, U_{2}, \ldots, U_{k}$ in $U$ each containing a component of $f^{-1}(H)$ and so that $f^{-1}(H) \cdot \sum \operatorname{Fr}\left(U_{i}\right)=0$. We may then apply the theorem in each $U_{i}$ and let $d(H)=\min d\left(H_{i}\right)$.

CoROllary 4. If $f$ is light and $y$ is a point of $B$ such that $f^{-1}(y)$ contains at least $k$ points, there exists $d(y)>0$ such that if $\rho(f, g)<d(y)$, then $g^{-1}(y)$ also contains at least $k$ points.

This is a special case $y=H$ of Corollary 3 , since each point of $f^{-1}(y)$ is a component of $f^{-1}(y)$ and a conditionally compact open set $U$ can be chosen in $A$ so as to contain at least $k$ points of $f^{-1}(y)$ and with $\operatorname{Fr}(U) \cdot f^{-1}(H)=0$.

COROLLARY $5\left({ }^{2}\right)$. Let the sequence $f_{n}(z)$ of functions each analytic in a region $D$ of the complex plane converge uniformly in $D$ to the nonconstant function $f(z)$ and let $z_{0}$ be a zero of $f(z)$. Then for any neighborhood $V$ of $z_{0}$ there exists an $N$ such that $V$ contains a zero of $f_{n}(z)$ for all $n>N$.

For since the mapping generated by $f$ is light we may choose $U \subset V$ so that $\operatorname{Fr}(U) \cdot f^{-1}(0)=0$, that is, so that $f(z) \neq 0$ on $\operatorname{Fr}(U)$. Then, taking $H=0$ (complex number) and applying the theorem, we have $\rho\left(f, f_{n}\right)<d(0)$ for $n>N$ and $f_{n}$ is strongly open so that $f_{n}(U) \supset 0$, that is, $f_{n}(z)=0$ for some $z \in U$.

4. Upper semi-continuity of degree. Preliminaries. It need hardly be remarked that Corollary 4 establishes the lower semi-continuity of the degree function as stated in $\$ 1$. To obtain upper semi-continuity, however, much stricter conditions must be imposed on the spaces involved. This may be indicated by a simple example. If $(\rho, \theta, z)$ are cylindrical coordinates in 3 -space $E_{3}$, let us define mappings

$$
\begin{aligned}
f(1, \theta, 0) & =(1,2 \theta, 0), \\
h_{\epsilon}(1, \theta, 0) & =\left(1-\epsilon \sin \theta, 2 \theta, \epsilon \sin \frac{\theta}{2}\right), \quad 0 \leqq \theta \leqq 2 \pi .
\end{aligned}
$$

Then $f$ is a "square" mapping of degree 2 of the unit circle $C$ of the $(\rho, \theta)$ plane onto itself. Whereas, for each $\epsilon>0, h_{\epsilon}$ is a homeomorphism of $C$ into $E_{3}$ satisfying

$$
\rho\left(f, h_{\epsilon}\right)<\epsilon(2)^{1 / 2} .
$$

(2) This is one form of the Hurwitz Theorem. See, for example, Titchmarsh, Theory of functions, p. 119. Other well known theorems on sequences of analytic functions follow similarly. For example, the result "if $f_{n}(z)$ is simple in a region $D$ for each $n$ and $f_{n}(z) \rightarrow f(z)$ uniformly in $D$, then either $f(z)$ is simple in $D$ or is a constant" (Titchmarsh, p. 200) follows directly from Corollary $\mathbf{5}$ as does also the fact that the limit of a uniformly convergent sequence of polynomials of degree less than or equal to $k$ is itself either constant or of degree less than or equal to $k$. 
Lemma $1\left(^{3}\right)$. Let $f(A)=B$ be light and open where $A$ and $B$ are closed 2-cells. If $f$ maps the edge $C$ of $A$ topologically onto the edge $C^{\prime}$ of $B$, then $f$ is a homeomorphism.

Proof. Suppose not. Then there exists a simple arc $\alpha=a^{\prime} y b^{\prime}$ in $B$ such that $C^{\prime} \cdot \alpha=a^{\prime}+b^{\prime}$, and $f^{-1}(y)$ contains two distinct points $x_{1}$ and $x_{2}$. Since each component of $f^{-1}(\alpha)$ maps onto $\alpha$ whereas $a^{\prime}$ and $b^{\prime}$ have unique inverses $a$ and $b$ respectively, $f^{-1}(\alpha)$ is connected. Thus $f^{-1}(\alpha)$ contains a simple arc $a x b$; and $f(a x b)=\alpha$ since $f(a x b) \subset \alpha$ and $f(a x b)$ contains an arc from $a^{\prime}$ to $b^{\prime}$. Since $B-\alpha$ consists of two components $R_{1}$ and $R_{2}$ each containing a component of $C^{\prime}-a^{\prime}-b^{\prime}$ and $\alpha \subset \operatorname{Fr}\left(R_{1}\right) \cdot \operatorname{Fr}\left(R_{2}\right), A-f^{-1}(\alpha)$ consists of exactly two components $Q_{1}$ and $Q_{2}$ containing the corresponding segments of $C-a-b$ and $f^{-1}(\alpha) \subset \operatorname{Fr}\left(Q_{1}\right) \cdot \operatorname{Fr}\left(Q_{2}\right)$. But since $a x b$ separates in $A$ these same two segments of $C-a-b$ and $a x b \subset f^{-1}(\alpha)$, we must have $a x b=f^{-1}(\alpha)$. Accordingly the mapping $f(a x b)=\alpha$ is open and $x_{1}+x_{2} \subset f^{-1}(y) \subset a x b$. This is impossible because $f$ must be topological on $a x b$ since $a=f^{-1}\left(a^{\prime}\right), b=f^{-1}\left(b^{\prime}\right)$.

Lemma 2. Let $f(J)=C$ be an open mapping of degrees $k>1$ where $J$ is a simple closed curve and $C$ is a circle of radius $r$ in a plane $Z$. Then for any homeomorphism $h: J \rightarrow Z$ whatever,

$$
\rho(f, h)>r / 4 .
$$

Proof. Suppose there is some $h$ with $\rho(f, h) \leqq r / 4$. Let $a$ and $b$ be diametrically opposite points on $C$ and designate the two arcs of $C$ by $a b$ and $b a$. Let $f^{-1}(a)=a_{1}+a_{2}+\cdots+a_{k}, f^{-1}(b)=b_{1}+b_{2}+\cdots+b_{k}$ with cyclic order $a_{1}, b_{1}, a_{2}, b_{2}, \cdots, a_{k}, b_{k}, a_{1}$ on $J$. Let $J^{\prime}=h(J)$ and, in general, $h(x)=x^{\prime}$ for $x \in J$. We have

$$
\sum a_{i}^{\prime} \subset V_{r / 4}(a), \quad \sum b_{i}^{\prime} \subset V_{r / 4}(b) .
$$

Consider the arcs

$$
a_{1}^{\prime} a_{2}^{\prime}, a_{2}^{\prime} a_{3}^{\prime}, \cdots, a_{k}^{\prime} a_{1}^{\prime}
$$

on $J^{\prime}$. These contain the points $b_{1}^{\prime}, b_{2}^{\prime}, \cdots, b_{k}^{\prime}$ respectively. There exists a linear segment $x y \subset V_{r / 4}(b)$ such that $x y \cdot J^{\prime}=x+y$ and $x$ and $y$ lie on different arcs of the set (2), say on $a_{1}^{\prime} x a_{2}^{\prime}$ and $a_{j}^{\prime} y a_{j+1}^{\prime}$. Let $K$ denote the simple closed curve formed by $a_{1}^{\prime} x+x y+y a_{j+1}^{\prime}+\sum_{j+1}^{k} a_{m}^{\prime} a_{m+1}^{\prime}$. Then $K=x y+\alpha$,

(3) It may be remarked that this lemma solves a sort of topological "boundary value problem." One may hazard the conjecture that any light open mapping of a closed $n$-cell $A$ onto an $n$-cell $B$ which maps the boundary of $A$ topologically on the boundary of $B$ is a homeomorphism. Lemma 2 is the case $n=2$ of this conjecture and it will be noted that its proof is based essentially on the case $n=1$. A slightly different form of Lemma 2 with a much less elementary proof is given on pp. 191-192 of the author's book Analytic topology, Amer. Math. Soc. Colloquium Publications, vol. 28, 1942. It includes the well known theorem that any function $w=f(z)$ analytic on and inside a simple closed curve $J$ of the complex plane which is simple on $J$ is also simple inside $J$ (see Titchmarsh, loc. cit., p. 201, for example). 
where $\alpha$ is one of the arcs of $J^{\prime}$ from $x$ to $y$.

Now since $a_{1}^{\prime} x+a_{j}^{\prime} y \subset V_{r / 4}(a b)$ and $x a_{2}^{\prime}+y a_{j+1}^{\prime} \subset V_{r / 4}(b a)$, it follows that $x a_{2}^{\prime}-x$ and $a_{j}^{\prime} y-y$ are separated by $K$. For taking $t$ as the midpoint of $a b$ we may find a linear segment $u v \subset V_{r / 4}(t)$ with $u \in a_{1}^{\prime} x, v \in y a_{j}^{\prime}$, and $u v$ $\cdot\left(a_{1}^{\prime} a_{2}^{\prime}+a_{j}^{\prime} a_{j+1}^{\prime}\right)=u+v$. Then since the simple closed curve $W=u x$ (of $\left.a_{1}^{\prime} a_{2}^{\prime}\right)+x y+y v$ (of $\left.y a_{j}^{\prime}\right)+v u$ together with its interior lies in $V_{r / 2}(t b)$, both $y a_{j+1}^{\prime}-y$ and $x a_{2}^{\prime}-x$ must be without $W$. The interior of $W$ lies entirely on one side of $K$ and this side contains $y a_{j}^{\prime}-y$ while $x a_{2}^{\prime}-x$ necessarily lies on the opposite side of $K$ since it lies without $W$. But this is impossible since then the open arc $J^{\prime}-\alpha$ of $J^{\prime}$ would have to intersect $K$ as it contains both $a_{2}^{\prime}$ and $a_{j}^{\prime}$.

CoROLlaRY. Let $f(J)=C$ be an open mapping of degree $k$ where $J$ is a simple closed curve and $C$ is a circle of radius $r$ on a plane $Z$. If there exists a homeomorphism $h: J \rightarrow Z$ satisfying $\rho(f, h)<r / 4$, then $k=1$ and $f$ is a homeomorphism.

5. Theorem. Let $f: A \rightarrow B$ be a compact light open mapping of degree $k$ where $A$ and $B$ are 2-manifolds. Then for any continuum $H \subset f(A)$ there exists an $e(H)>0$ such that if $g: A \rightarrow B$ is any light strongly open mapping satisfying $\rho(f, g)<e(H)$, then $g$ is compact and of degree $k$ on $g^{-1}(H)$.

Proof. We may suppose without loss of generality that $H$ contains interior points. Then we may choose an interior point $y$ of $H$, which we first suppose is the center of a circular disc $E$ of radius $4 r$ lying wholly in $H$, such that $f^{-1}(E)$ consists of $k$ disjoint topological discs $E_{1} \cdots E_{k}$ each of which maps topologically onto $E$ under $f$. Let $D$ be the circular disc centered at $y$ and of radius $r$ and let $E_{i} \cdot f^{-1}(D)=D_{i}$. Let $C$ be the circle centered at $y$ and of radius $2 r$, let $R$ be its interior and $S$ its exterior relative to the interior of $E$. Determine $d(H)>0$ as in $\S 3$, Corollary 2 and define $e(H)=\min [r / 2, d(H)]$. Now let $g: A \rightarrow B$ satisfy the conditions in our theorem. Let $J_{i}=g^{-1}(C) \cdot E_{i}$.

Then since $\rho\left[g\left(D_{i}\right), f\left(D_{i}\right)\right]=\rho\left[g\left(D_{i}\right), D\right]<r / 2, g\left(D_{i}\right)$ is within $C$ so that $D_{i}$ lies in a bounded component $Q_{i}$ of the complement of $J_{i}$ relative to the interior of $E_{i}$. Since any bounded component $Q_{i}$ of the complement of $J_{i}$ (rel. int. $E_{i}$ ) must map onto $R$ under $g$, there can be only one such in $E_{i}$, because a second one could contain no point of $f^{-1}(y)$ whereas it would contain a point $x$ of $g^{-1}(y)$ and we would have $\rho[f(x), g(x)]=\rho[f(x), y]>r$. Likewise there can be only one unbounded component $G_{i}$ of the complement of $J_{i}$ (rel. int. $\left.E_{i}\right)$; and since $J_{i} \subset \operatorname{Fr}\left(Q_{i}\right) \cdot \operatorname{Fr}\left(G_{i}\right)$, and $J_{i}$ is locally connected, it follows that $J_{i}$ is a simple closed curve.

Next consider the mappings $h=f \mid J_{i}$ and $g_{i}=g \mid J_{i}$. Since $h$ is a homeomorphism, $g_{i}\left(J_{i}\right)=C$ is open and $\rho\left(h, g_{i}\right)<e(H) \leqq 2 r / 4$, it follows by $\S 4$, Lemma 2 that $g_{i}$ is a homeomorphism. But then since $J_{i}=\left(Q_{i}+J_{i}\right) \cdot g^{-1}(C)$, so also is the mapping $g \mid\left(Q_{i}+J_{i}\right)$ by Lemma 1 . Finally, since for any $x$ $\in\left[g^{-1}(H)-\sum_{1}^{k}\left(Q_{i}+J_{i}\right)\right]$, the point $f(x)$ lies in $B-D$, we have $\rho[g(x), B-D]$ 
$<r$ so that $g(x) \neq y$. Thus $g^{-1}(y) \subset \sum\left(Q_{i}+J_{i}\right)$ and thus $g^{-1}(y)$ consists of exactly $k$ points at each of which $g$ is locally topological. Thus $g$ is of degree $k$ on $g^{-1}(H)$.

In the proof just given it was assumed that $y$ is the center of a circular $\operatorname{disc} E$ in $H$. To complete the proof let $t(B)=B^{\prime}$ be a topological mapping where $B^{\prime}$ is a manifold such that $t(y)$ is the center of a circular disc on $t(H)$. By the case already proven, there is an $e^{\prime}(H)>0$ such that $\rho(t f, t g)<e^{\prime}(H)$ gives deg. $t g=k$ on $g^{-1}(H)$. Let $e(H)>0$ be determined so that $\rho(x, y)<e(H)$, $x, y \in H$, gives $\rho[t(x), t(y)]<e^{\prime}(H)$. Then if $\rho(f, g)<e(H)$, we have $\rho(t f, t g)$ $<e^{\prime}(H)$ and deg. $t g=$ deg. $g=k$ on $g^{-1}(H)$.

\section{Application.}

TheOREM ON THE "Zeros." Let $A$ and $B$ be 2-manifolds and $f: A \rightarrow B$ be light and strongly open. Let $D$ be a conditionally compact region in $A$ with boundary $C$ and let $y \in[f(D)-f(C)]$. There exists an $e(y)>0$ such that any light strongly open mapping $g: A \rightarrow B$ satisfying $\rho(f, g)<e(y)$ has the same number of $y$-places in $D$ (each counted with multiplicity) as does $f$.

Proof. We let $B^{*}$ be the component of $B-f(C)$ containing $y$ and let $A^{*}$ $=D \cdot f^{-1}\left(B^{*}\right)=A_{1}+\cdots+A_{m}$ (components). Consider the mappings

$$
f \mid A_{j}=f_{j}, \quad f_{j}\left(A_{j}\right)=B^{*} \subset B, \quad j=1, \cdots, m .
$$

By $\S 2$, each of these is compact, light, and strongly open and of degree $k_{j}$ and $\sum k_{j}=k$ equals the number of $y$-places of $f$ in $D$. For each $j$, let $e_{j}(y)$ be the number given by $\$ 5$ for the mapping $f_{j}$ and define

$$
e(y)=\min \left[e_{j}(y), \rho\left(y, B-B^{*}\right)\right] .
$$

Then if $g: A \rightarrow B$ satisfies the condition $\rho(f, g)<e(y)$, it follows by $\S 5$ that $g \mid A_{j}$ is of degree $k_{j}$ for each $j$. Further, for any $x \in D-A^{*}$,

$$
p(g(x), f(x))<e(y) \leqq \rho\left(y, B-B^{*}\right) \leqq \rho(y, f(x))
$$

since $f(x) \in B-B^{*}$. Thus $g(x) \neq y$ so that

$$
g^{-1}(y) \cdot D \subset A^{*}
$$

Accordingly $g$ takes the value $y$ exactly $k=\sum k_{j}$ times (each counted with multiplicity) in $D$.

COROLLARY. The same conclusion holds for each $y_{0}$ in some neighborhood of $y$ with $k$ constant.

As a second corollary we have the following theorem.

HURWITZ THEOREM. Let the sequence of functions $f_{n}(z)$ each analytic in a region $R$ converge uniformly in $R$ to a function $f(z)$ which is not identically zero. Then if $\zeta \in R$ is an $m$-fold zero of $f(z)$, every sufficiently small neighborhood $D$ 
of $\zeta$ contains exactly $m$ zeros of $f_{n}(z)$ for $n>N(D)$.

For since $f(z)$ is nonconstant and analytic in $R$ its mapping of $R$ into the $w$-plane is light and strongly open. Thus if we take $D+C$ in $R$ with $\zeta \in D$ so that $f(z) \neq 0$ on $D+C-\zeta$ and obtain $e(0)$ from the theorem, our conclusion holds for each $f_{n}$ satisfying $\rho\left(f, f_{n}\right)<e(0)$.

UNIVERSITY OF VIRGINIA, Charlottesville, Va. 